\title{
Manipulación del transporte y consumo de oxígeno en la sepsis
}

\author{
TOMAS REGUEIRA, MAX ANDRESEN
}

\section{Management of oxygen delivery and consumption during sepsis}

Maintaining an adequate tissue oxygen delivery $\left(\mathrm{DO}_{2}\right)$ and consumption $\left(\mathrm{VO}_{2}\right)$ is crucial in the treatment of septic patients. A fall in $\mathrm{VO}_{2}$ is associated with a higher mortality. The early recognition of shock or tissue hypoperfusion impacts on patient prognosis. In occasions, hypovolemia or important regional oxygen debts are not recognized, since macro hemodynamic variables have been compensated. In this situation, the use of metabolic hypoperfusion markers such as lactate, central venous oxygen saturation and gastric tonometry, can be helpful. However, interpretation of these markers should be cautious and always considering the overall clinical status of the patient. In the initial stages of sepsis, the dependency of $\mathrm{VO}_{2}$ on $\mathrm{DO}_{2}$ predominates as physiopathological mechanism of multiple organic failure. In late stages, other factors predominate as determinants of multiple organic failure and mortality, such as hyper or hypo immune response, microcirculatory alterations and cytopathic hypoxia.

(Rev Med Chile 2010; 138: 233-242).

Key words: Anoxia; Sepsis; Shock.

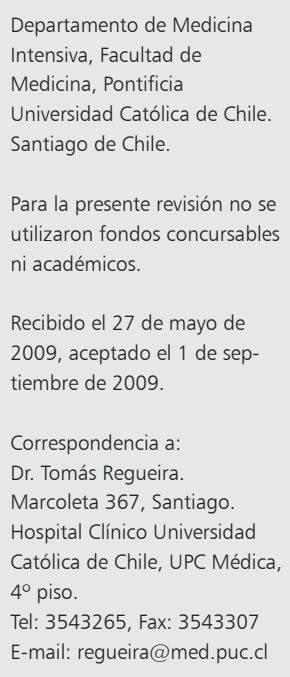

L a mortalidad por sepsis continúa siendo alta $^{1,2}$ y su prevalencia significativa ${ }^{1}$. Para la sepsis severa la tasa de mortalidad alcanza hasta $28-32 \%$, mientras que en el shock séptico, ésta llega a valores de 50-60\% (Tabla 1). Existe una relación directa entre el desarrollo del síndrome de disfunción multiorgánica (SDMO) y mortalidad; así, pacientes sépticos con disfunción de 4 o más sistemas alcanzan mortalidades de $65 \%{ }^{2}$. Los mecanismos responsables del desarrollo de SDMO durante la sepsis permanecen aún en estudio $^{3-5}$. En esta revisión se discute el rol del transporte y consumo de oxígeno, y estrategias para su optimización, como aspecto fundamental en el tratamiento de enfermos sépticos.

\section{Transporte del oxígeno $\left(\mathrm{DO}_{2}\right)$}

El objetivo del sistema cardio-pulmonar es llevar el oxígeno presente en la atmósfera hasta las mitocondrias de cada célula del organismo. La presión parcial de oxígeno a nivel del mar es de aproximadamente $159 \mathrm{mmHg}(760 \mathrm{mmHg} \times 0,21)$, desde esta presión inicial, la cascada del oxígeno es tan abrupta, que al llegar a la mitocondria lo hace con presiones parciales de 4 a $8 \mathrm{mmHg}$ (Figura 1). La hemoglobina $(\mathrm{Hb})$ es el principal transportador de oxígeno en la sangre; cada gramo de $\mathrm{Hb}$ transporta $1,34 \mathrm{ml} \mathrm{de} \mathrm{O}_{2}$. El contenido arterial de oxígeno $\left(\mathrm{CaO}_{2}\right)$ depende fundamentalmente de la concentración de $\mathrm{Hb}$ y de su saturación y puede calcularse como: $\mathrm{CaO}_{2}=\left(1,34 \times \mathrm{Hb} \times\left(\mathrm{SaO}_{2} / 100\right)\right)$ $+\left(0,003 \times \mathrm{PaO}_{2}\right)$. El producto del $\mathrm{CaO}_{2}$ por el gasto cardíaco (GC) nos permite estimar el $\mathrm{DO}_{2}:\left(\mathrm{DO}_{2}\right.$ $\left.=\mathrm{CaO}_{2} \times \mathrm{GC}\right)$.

La saturación de $\mathrm{Hb}\left(\mathrm{SaO}_{2}\right)$ en la sangre está determinada por la curva de disociación de la $\mathrm{Hb}$, que compara la $\mathrm{PaO}_{2}$ con la $\mathrm{SaO}_{2}$ (Figura 2). La curva se comporta de tal manera que bajo una $\mathrm{SaO}_{2}$ de $90 \%$, pequeños cambios en $\mathrm{la}_{\mathrm{PaO}_{2}}$ se asocian a grandes cambios en la $\mathrm{SaO}_{2}$. En general 
Tabla 1. Definiciones de la sepsis (ACCP/SCCM consensus conference, Chest 1992; 101: 1644)

\section{Sepsis}

a. Infección documentada o sospechada +

b. SIRS

i. Fiebre $\left(\mathrm{T}^{\circ} \geq 38,13^{\circ} \mathrm{C}\right)$ o hipotermia $\left(\mathrm{T}^{\circ} \leq 36^{\circ} \mathrm{C}\right)$.

ii. Frecuencia cardíaca $>90 \mathrm{lpm}$

iii. Frecuencia respiratoria $>20 \mathrm{rpm}$

iv. Leucocitosis $>12.000$ o leucopenia $<4.000$ o formas inmaduras $>10 \%$.

\section{Sepsis severa}

a. Sepsis +

b. Disfunción de órganos

i. $\mathrm{PaO}_{2} / \mathrm{FiO}_{2}<300$

ii. $\uparrow$ creatinina $>0,5 \mathrm{mg} / \mathrm{dl}$ u oliguria

iii. Alteraciones de coagulación (INR $>1,5$ o TTPK $>60$ seg)

iv. Trombocitopenia

v. Hiperbilirrubinemia

vi. $l l e o$

c. Hipoperfusión

i. Hiperlactacidemia

ii. Oliguria

iii. Alteración del estado de conciencia

\section{Shock séptico}

a. Sepsis severa +

b. Hipotensión arterial persistente (PAS $<90$ o PAS que baja $>40$ $\mathrm{mmHg}$ de su basal) a pesar de una resucitación con fluidos adecuada y que requiere inicio de vasopresores.

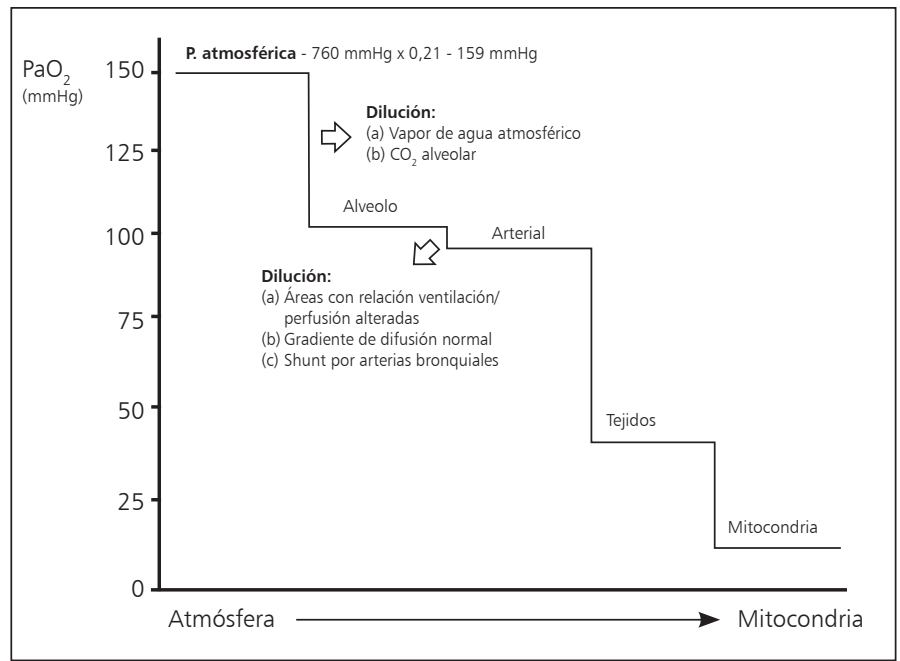

Figura 1. Cascada del oxígeno. La respiración es un fenómeno celular, la presión parcial de oxígeno intracelular debe ser mantenida entre $5-8 \mathrm{mmHg}$. una $\mathrm{SaO}_{2}$ de $50 \%$ se asocia a una $\mathrm{PaO}_{2}$ de 26,6 mmHg (Figura 2).

Una vez en los tejidos, parte de las moléculas de oxígeno pasan al espacio intersticial, difundiendo libremente al espacio intracelular y a las mitocondrias, donde es utilizado como aceptor final de electrones en el complejo IV de la cadena de fosforilación oxidativa mitocondrial. La cantidad de oxígeno que se libera en cada tejido es proporcional a sus demandas metabólicas y a su capacidad de extracción, determinando una presión venosa de oxígeno $\left(\mathrm{PvO}_{2}\right)$ particular para cada tejido.

\section{Eventos hemodinámicos y metabólicos relacionados con el transporte $\left(\mathrm{DO}_{2}\right)$ y consumo $\left(\mathrm{VO}_{2}\right)$ de oxígeno durante la sepsis}

La sepsis se caracteriza por una marcada hipovolemia efectiva, secundaria a un aumento de la permeabilidad capilar, que produce paso de volumen desde el espacio intravascular a los tejidos y a una baja significativa de las resistencias vasculares, por vasodilatación tanto arteriolar como venosa; contribuyen además a la hipovolemia la disminución de ingesta y el aumento de pérdidas insensibles. Se genera así una redistribución del flujo sanguíneo, con reducción del mismo al miocardio, músculo esquelético, estómago, duodeno e intestino y al páncreas ${ }^{6,7}$. Son también fenómenos propios de la sepsis, la obstrucción microvascular y la disfunción miocárdica; ésta se caracteriza por depresión de la contractibilidad biventricular sistólica y disminución de la distensibilidad ventricular diastólica ${ }^{8}$. Los factores mencionados, entre otros, se asocian a una caída en el $\mathrm{DO}_{2}$ sistémico y regional.

Por otro lado, la sepsis se caracteriza también por hipermetabolismo, con aumento del catabolismo protei- 


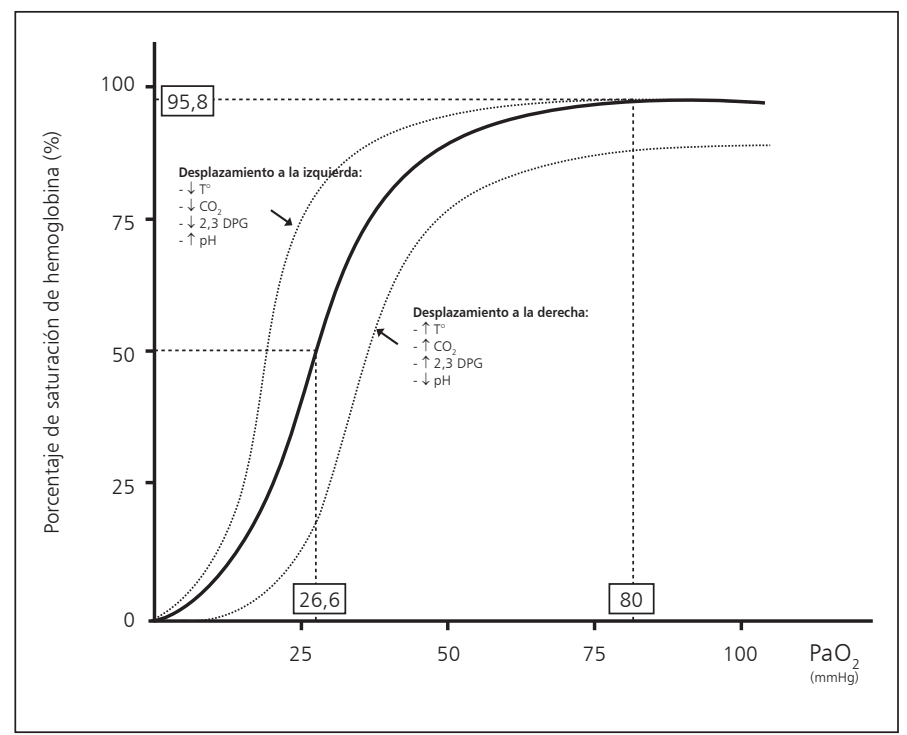

Figura 2. Curva de disociación de la hemoglobina.

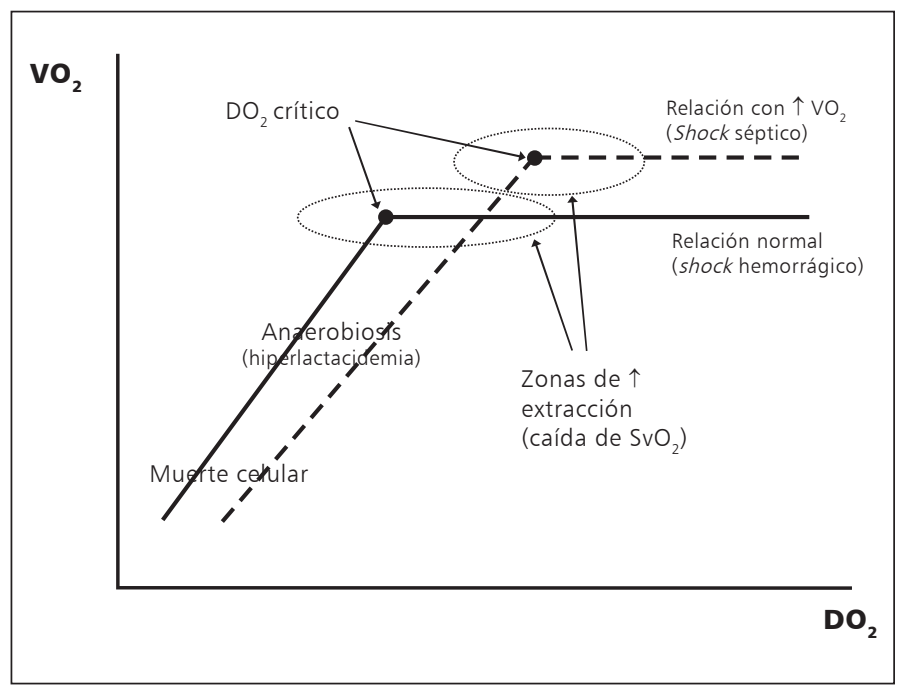

Figura 3. Grafica que compara la relación existente entre transporte de oxígeno $\left(\mathrm{DO}_{2}\right)$ y consumo de oxígeno $\left(\mathrm{VO}_{2}\right)$. En individuos normales (línea continua) la relación $\mathrm{DO}_{2} \mathrm{NO}_{2}$ sigue una relación bifásica, en etapas iniciales una caída progresiva en el $\mathrm{DO}_{2}$ no afecta el $\mathrm{VO}_{2}$, inicialmente por un amplio margen de reserva en el DO2 respecto a las demandas metabólicas de reposo y luego por un aumento en la extracción de oxígeno. Alcanzado un punto crítico, la capacidad de extracción se satura y el $\mathrm{VO}_{2}$ se hace dependiente del $\mathrm{DO}_{2}$, iniciándose metabolismo anaeróbico y acumulación de lactato. En individuos sépticos (línea intermitente) se cumple la misma relación bifásica, pero dado que existe hipermetabolismo, el $\mathrm{VO}_{2}$ se encuentra aumentado, y el punto critico de dependencia $\mathrm{DO}_{2} \mathrm{NO}_{2}$, a mayores valores de $\mathrm{DO}_{2}$. co, de la oxidación de aminoácidos ramificados, de la síntesis de proteínas de fase aguda y de la gluconeogénesis hepática. El hipermetabolismo de la sepsis condiciona un aumento en el gasto energético y en el $\mathrm{VO}_{2}$ global ${ }^{9}$.

La caída en el $\mathrm{DO}_{2}$ global y regional propia de la sepsis, asociado al aumento en el $\mathrm{VO}_{2}$ descrito, genera una deuda de oxígeno tisular. Esta deuda de oxígeno se caracteriza por una dependencia directa del $\mathrm{VO}_{2}$ al $\mathrm{DO}_{2}$, la que actuaría como un elemento central en la génesis del SDMO durante las etapas precoces de la sepsis (Figura 3).

\section{Marcadores metabólicos de hipo- perfusión durante la sepsis}

La persistencia de una deuda de oxígeno global o regional es una de las causas que favorecen el desarrollo del SDMO durante la sepsis. Desde un punto de vista clínico es fundamental en el manejo de la sepsis la capacidad de reconocer el shock y la hipoperfusión tisular precozmente. Sin embargo, pacientes con hipovolemia o deudas de oxígeno regionales persistentes pueden no ser diagnosticados correcta y oportunamente como shock, puesto que a menudo han compensado las variables macro-hemodinámicas comúnmente medidas.

Durante la última década se ha puesto énfasis en la búsqueda de marcadores más sensibles y específicos de oxigenación tisular. El uso de variables metabólicas como el lactato, la saturación venosa central de oxígeno, la tonometría gástrica, entre otros, se ha sugerido como una manera de optimizar la sensibilidad a la presencia de hipoperfusión persistentes.

\section{a) Hiperlactacidemia}

Los niveles de lactato en el plasma se correlacionan con mortalidad en los pacientes sépticos y su aclaramien- 
to precoz se asocia a mejoría en la sobrevida ${ }^{10,11}$. La elevación de los niveles de lactato plasmáticos se ha asociado tradicionalmente a metabolismo anaeróbico por un insuficiente $\mathrm{DO}_{2}$ para los requerimientos tisulares, dato que se ha usado para identificar aquellos pacientes que pudiesen beneficiarse de una optimización de su DO. A la fecha no existen trabajos prospectivos publicados que hayan estudiado la reanimación de pacientes sépticos usando el lactato como meta de reanimación.

Una publicación reciente sugiere que la hiperlactacidemia de la sepsis podría no ser sólo secundaria a hipoperfusión, sino también a trastornos del metabolismo celular no hipóxico ${ }^{12}$, por lo que su interpretación debe ser cautelosa, particularmente en la sepsis reanimada.

El lactato es producido en el citoplasma a partir de piruvato en una reacción mediada por la enzima lactato deshidrogenasa. Esta reacción favorece la formación de lactato, con una relación fisiológica de lactato: piruvato (L:P) de 10:1. El piruvato es el producto final de la glicólisis y normalmente entra en la mitocondria y es usado como sustrato en el ciclo de Krebs del metabolismo oxidativo. Durante períodos de bajo $\mathrm{DO}_{2}$ tisular existe bloqueo de la síntesis mitocondrial de ATP (cae la relación ATP/ ADP) y aumentan los niveles de NADH (aumenta la relación NADH/NAD). Ambos fenómenos se asocian a acumulación de piruvato por bloqueo de su metabolismo y favorecen la transformación de piruvato en lactato, aumentando la relación L:P. La hipoperfusión se asocia a hiperlactacidemia con una relación L:P aumentada, alta utilización de glucosa por la vía glicolítica y baja producción de energía. Niveles elevados de la relación L:P se encuentran en pacientes con shock cardiogénico, en shock séptico refractario que requieren altas dosis de catecolaminas, pero no en pacientes o en modelos de sepsis o shock séptico estabilizados o con $\mathrm{pH}$ normal ${ }^{13}$. De hecho, en la sepsis reanimada, el aumento del $\mathrm{DO}_{2}$ no corrige la hiperlactacidemia ${ }^{14}$, los niveles de $\mathrm{PO}_{2}$ y ATP tisulares no están disminuidos ${ }^{15,16}$ y no parece existir producción regional de lactato hepato-esplácnica ${ }^{17}$. Visto así, el origen del lactato en la sepsis reanimada parece ser metabólico. Uno de los mecanismos metabólicos propuestos para explicar el aumento del lactato durante la sepsis es la activación de dos vías glicolíticas paralelas, una de las cuales provee de ATP a la bomba $\mathrm{Na}^{+} / \mathrm{K}^{+}$-ATPasa de membrana, y es particularmente sensible a la estimulación por catecolaminas sobre receptores $\mathrm{B}_{2}$ de membrana ${ }^{18}$. El bloqueo selectivo de la bomba $\mathrm{Na}^{+} / \mathrm{K}^{+}$-ATPasa muscular en forma local durante la sepsis, se asocia a inhibición en la producción de lactato, sugiriendo la participación de un componente metabólico, no asociado a hipoperfusión, en la hiperlactacidemia de la sepsis ${ }^{19}$.

\section{b) Tonometría gástrica}

La perfusión esplácnica persistentemente inadecuada se ha asociado con un mayor riesgo de desarrollar el $\mathrm{SDMO}^{20}$. La circulación esplácnica aparece como una zona particularmente sensible a la sepsis. Tanto los flujos principales, como la irrigación terminal de la mucosa se ven afectadas durante la sepsis, incluso con parámetros macrohemodinámicos normales ${ }^{21}$. Reducciones de flujo mayores de $50 \%$ vuelven al $\mathrm{VO}_{2}$ de la mucosa esplácnica $\mathrm{DO}_{2}$ dependiente ${ }^{22}$. La reperfusión del intestino también produce lesión mediada por radicales libres.

El principio de la tonometría se basa en que durante los períodos de hipoperfusión se produce acumulación de $\mathrm{CO}_{2}$ en la mucosa gástrica e intestinal. El tonómetro consta de una sonda oro o nasogástrica con un balón en el extremo distal que se infla con solución salina o aire; el $\mathrm{CO}_{2}$ acumulado en la mucosa difunde fácilmente a través de la membrana del balón, igualándose las $\mathrm{PCO}_{2}$ de la mucosa con la de la luz del balón. El $\mathrm{CO}_{2}$ asî recogido es medido por el tonómetro $\left(\mathrm{PrCO}_{2}\right)$. Un aumento en la diferencia entre del $\mathrm{PrCO}_{2} \mathrm{y}$ $\mathrm{PaCO}_{2}$ o $\mathrm{PeCO}_{2}$ (presión parcial de $\mathrm{CO}_{2}$ espirado medido por capnografía $)^{23}$ son indicadores de hipoperfusión tisular.

La tonometría gástrica ha mostrado ser un buen predictor de complicaciones y mortalidad en pacientes $\operatorname{críticos}^{24}$, en pacientes en proceso de "destete" de ventilación mecánica ${ }^{25}$, en pacientes con trauma ${ }^{20}$ e hipertensión intraabdominal ${ }^{26} \mathrm{y}$ especialmente, en pacientes sépticos. Aun así, no ha demostrado ser útil en disminuir la mortalidad, al utilizarse como meta de reanimación.

\section{c) Saturación venosa central de oxígeno $\left(\mathrm{SvO} \mathrm{O}_{2}\right)$}

La presión parcial de oxígeno con que la sangre venosa retorna al corazón $\left(\mathrm{PvO}_{2}\right)$ determina una saturación venosa de oxígeno (valores normales 65-70\%). $\mathrm{La} \mathrm{SvO}_{2}$ depende de la sumatoria de las extracciones de oxígeno individuales de cada tejido, la que depende a su vez de su metabolismo 
y del flujo sanguíneo regional respectivo (Figura 4). La corrección precoz de la $\mathrm{SvO}_{2}$, mediante la optimización del $\mathrm{DO}_{2}$, como objetivo terapéutico en la sepsis ha demostrado disminuir la aparición del SDMO y la mortalidad ${ }^{27}$. En contraste, la corrección tardía de la $\mathrm{SvO}_{2}$ no se ha asociado a estos beneficios $^{28}$ (ver más adelante).

\section{Optimización e interpretación del $\mathrm{DO}_{2} \mathrm{y} \mathrm{VO}_{2}$ en la sepsis}

Durante la década 1980-89, y hasta la fecha, la optimización del $\mathrm{DO}_{2}$ ha sido la variable de mayor relevancia en el manejo de los enfermos sépticos. Shoemaker fue uno de los pioneros en el estudio de la deuda de oxígeno como factor determinante de mortalidad y $\mathrm{SDMO}^{29,30}$. Estudiando enfermos quirúrgicos de alto riesgo observó que durante el período operatorio y postoperatorio de estos pacientes existía frecuentemente una deuda de oxígeno estimada como la diferencia entre el consumo esperado y el medido, y que esta deuda se correlacionaba a mortalidad y aparición de falla de órganos. Observó también que mientras mayor fuera el tiempo que permaneciera el paciente en deuda, mayor era su riesgo de desarrollar complicaciones $^{30}$. Posteriormente, este mismo autor estudió aleatoriamente en forma prospectiva pacientes quirúrgicos de alto riesgo a manejo estándar o a un grupo cuyo objetivo fue la optimización macro-hemodinámica pre-operatoria que se caracterizaba por un GC $>4,5 \mathrm{~L} / \mathrm{min} \cdot \mathrm{m} 2$, un $\mathrm{DO}_{2}>600 \mathrm{ml} / \mathrm{min} \cdot \mathrm{m}^{2}$ y un $\mathrm{VO}_{2}>170 \mathrm{ml} / \mathrm{min} \cdot \mathrm{m} 2$. Los pacientes del grupo intervenido presentaron una disminución de su mortalidad, de las complicaciones y de la estadía en la unidad de cuidados intensivos (UCI) ${ }^{29}$. También en la década 1980-89, otros grupos estudiaron la existencia de deuda de oxígeno en la sepsis y el shock séptico y observaron su relevancia en el desarrollo de complicaciones. Los grupos de Astiz y Kaufman ${ }^{31,32}$ mostraron que, efectivamente en pacientes con shock séptico hiperdinámico, el aumento del $\mathrm{DO}_{2}$ se asociaba a aumento significativo del $\mathrm{VO}_{2}$ y a descenso en los niveles de lactato, sugiriendo que el consumo de oxígeno en la sepsis estaba limitado por una perfusión limitada, estos grupos postularon que el concepto de deuda de oxígeno se empleaba también en la sepsis. Por otro lado, Gilbert y cols ${ }^{14}$ mostraron que sólo los pacientes con hiperlactacidemia respondían aumentando su $\mathrm{VO}_{2}$ frente al

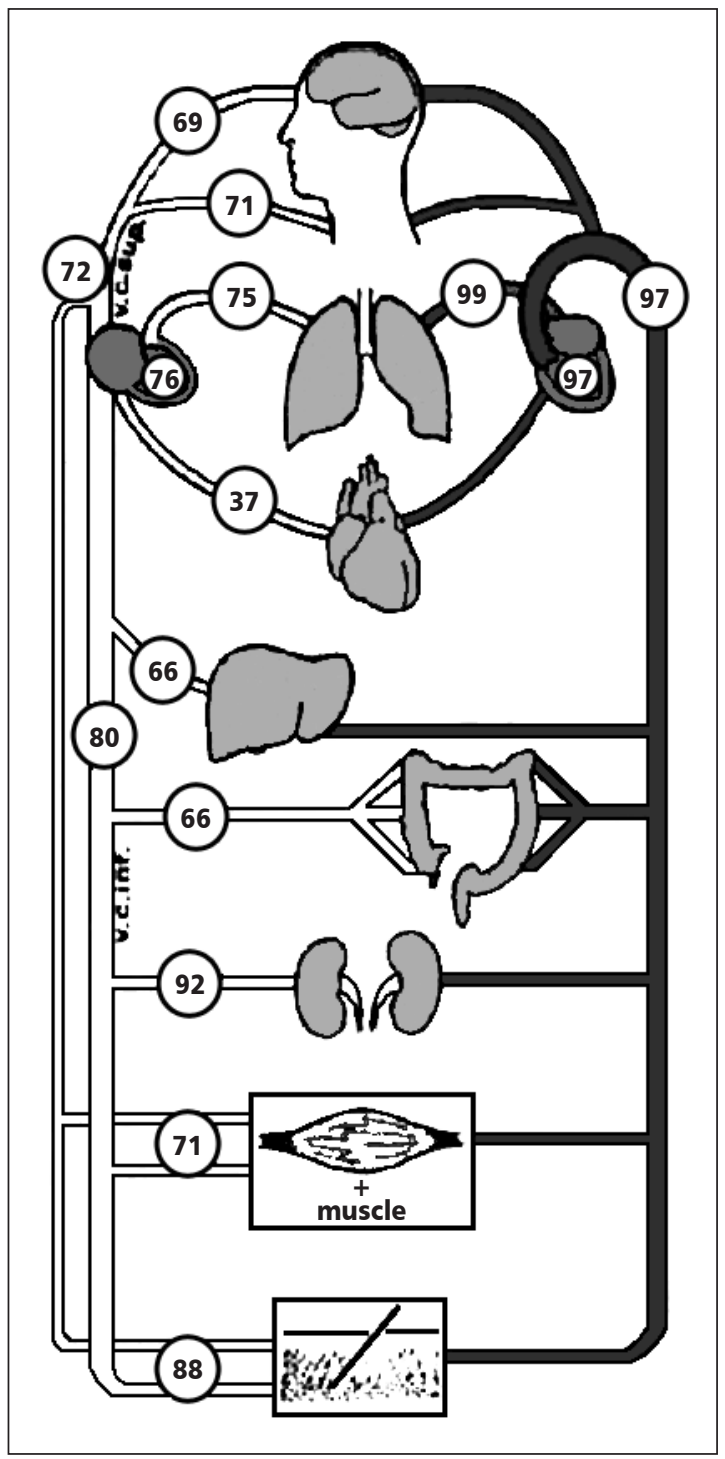

Figura 4. Saturaciones venosas promedio individuales de cada tejido.

uso de coloides o transfusiones para aumentar el $\mathrm{DO}_{2}$ y que el $\mathrm{VO}_{2}$ aumentaba también con el uso de dopamina o dobutamina independientemente de la presencia de hiperlactacidemia; sugiérese por tanto, que las drogas catecolaminérgicas tienen un efecto directo sobre el metabolismo oxidativo y que el $\mathrm{VO}_{2}$ de los pacientes sépticos puede cambiar también en relación a maniobras terapéuticas como las drogas vasoactivas (efecto termogénico). Se colige, entonces, que la base fisiopatológica central del SDMO se funda en la persistencia de deuda 
de oxígeno que presentan los pacientes críticos sépticos, sosteniéndose así la práctica generalizada de aumentar u optimizar el $\mathrm{DO}_{2}$ como eje de la prevención y tratamiento de SDMO.

En la década 1990-99, varios estudios prospectivos buscaron determinar si la optimización del $\mathrm{DO}_{2}$ disminuía la incidencia de SDMO y su mortalidad. Uno de los primeros trabajos es de Reinhart $^{33}$, quien aumentó significativamente el $\mathrm{DO}_{2}$ en pacientes críticos, lo cual se asoció a un aumento de sólo $10 \%$ en el $\mathrm{VO}_{2}$ y este cambio fue similar tanto en sobrevivientes, como en fallecidos ("no-sobrevivientes"). Reinhart atribuyó el escaso impacto al adecuado soporte entregado a los pacientes previo a la intervención. Gattinoni ${ }^{28}$, en 1995, publicó un estudio prospectivo, multicéntrico, en el cual distribuyó aleatoriamente 762 pacientes críticos, con menos de 48 horas de UCI, a tres grupos. El primero a un índice cardíaco "normal" entre 2,5 y $3 \mathrm{~L} / \mathrm{min} \cdot \mathrm{m}^{2}$, el segundo a un índice cardíaco "supra-normal" sobre $4,5 \mathrm{~L} /$ $\mathrm{min} \cdot \mathrm{m}^{2}$ y a un tercer grupo, a normalizar su $\mathrm{SvO}_{2}$ a valores mayores o iguales de $70 \%$. El estudio no mostró diferencias significativas en mortalidad intra UCI ni a los 6 meses post alta. Otro estudio, de Hayes $^{34}$, sometió aleatoriamente 109 pacientes críticos a reanimación estándar o a reanimación supra-máxima, mediante el uso de volumen y dobutamina, con objetivos hemodinámicos de IC $>4,5 \mathrm{~L} / \mathrm{min} \cdot \mathrm{m}^{2}, \mathrm{DO}_{2}>600 \mathrm{ml} / \mathrm{min} \cdot \mathrm{m}^{2}$ y un $\mathrm{VO}_{2}>$ $170 \mathrm{ml} / \mathrm{min} \cdot \mathrm{m}^{2}$. Hayes observó que a pesar de un significativo aumento en el $\mathrm{DO}_{2}$ en grupo intervenido, el $\mathrm{VO}_{2}$ no fue diferente entre los dos grupos y aun más, la mortalidad intra-hospitalaria fue significativamente mayor en el grupo intervenido ( $56 \%$ vs $34 \%, p=0,04)$, sugiriendo que el empleo de una reanimación supra-máxima pudiese ser incluso deletéreo.

La optimización del $\mathrm{DO}_{2}$ a niveles supra-normales también fue estudiada en forma específica en pacientes con sepsis y shock séptico. Inicialmente, Tuchschmidt ${ }^{35}$, en el año 1992 distribuyó aleatoriamente 51 pacientes en shock séptico con hiperlactacidemia a tratamiento estándar (IC $\geq 3 \mathrm{~L} / \mathrm{min} \cdot \mathrm{m}^{2}$ ) o a reanimación supra-máxima (IC $\geq 6 \mathrm{~L} / \mathrm{min} \cdot \mathrm{m}^{2}$ ). El grupo intervenido mostró una tendencia no significativa hacia una mejoría en la sobrevida $(50 \%$ vs $72 \%, \mathrm{p}=0,14)$ y a una disminución del tiempo de estadía en UCI. Sin embargo, en 1999 el grupo español encabezado por Inmaculada Alía ${ }^{36}$ trató aleatoriamente a 63 pacientes con sepsis severa y shock séptico a reanimación estándar o supra-normal $\left(\mathrm{DO}_{2}>600\right.$ $\left.\mathrm{ml} / \mathrm{min} \cdot \mathrm{m}^{2}\right)$. En este estudio sólo $9 / 31$ pacientes en el grupo intervenido lograron efectivamente un $\mathrm{DO}_{2}>600 \mathrm{ml} / \mathrm{min} \cdot \mathrm{m}^{2}$, no logrando establecer diferencias significativas entre los grupos en $\mathrm{DO}_{2}$. Tanto el $\mathrm{VO}_{2}$, como la mortalidad y el número de disfunciones de órganos fueron similares y sin diferencias entre los grupos.

Los estudios presentados, tanto en enfermos críticos generales como en pacientes sépticos comparten algunas características: Todos fallan en demostrar que el uso de una meta de reanimación basada en aumentar el transporte de oxígeno a valores supra-fisiológicos mejore la sobrevida de los pacientes. Todos fueron realizados en pacientes que ya llevaban hasta 48 horas en UCI y todos, salvo el grupo que se observó contra $\mathrm{SvO}_{2}$ en el trabajo de Gattinoni, carecen de metas de reanimación que integren variables metabólicas además de las puramente hemodinámicas.

En el año 2001, Rivers ${ }^{27}$ recogió la experiencia anterior y distribuyó en forma aleatoria precozmente 263 pacientes con sepsis severa en la unidad de urgencias a reanimación estándar versus reanimación agresiva, estrategia basada fundamentalmente en la corrección de los valores de $\mathrm{SvO}_{2}$, mediante el uso de volumen, glóbulos rojos y dobutamina. Efectivamente el grupo intervenido en el estudio de Rivers recibió durante las primeras 6 horas más volumen $(3500 \mathrm{ml}$ vs 4981 $\mathrm{ml})$, más transfusiones $(18,5 \%$ vs $64,1 \%)$ y ocupó más dobutamina $(0,8 \%$ vs $13 \%)$. La intervención se asoció efectivamente a mayores valores de $\mathrm{SvO}_{2}$ durante las primeras 72 horas, menores valores de lactato y mejor $\mathrm{pH}$. El grupo intervenido tuvo una significativa disminución en la aparición de falla multiorgánica y en la mortalidad hospitalaria (30,5\% vs $46,5 \%, \mathrm{p}=0,009)$.

El estudio de Rivers, y su comparación con los trabajos anteriores, permitió el replanteamiento de varios postulados en el tema de la manipulación del $\mathrm{DO}_{2} \mathrm{y} \mathrm{VO}_{2}$ en los pacientes sépticos. En primer lugar, pareciera que se pueden distinguir dos fases o tiempos en la génesis del SDMO y la mortalidad asociada a éste en los pacientes sépticos. Una primera fase precoz dependiente del transporte de oxígeno, en la cual la optimización del $\mathrm{DO}_{2}$ para mantener una $\mathrm{SvO}_{2}$ adecuada, vale decir un $\mathrm{DO}_{2}$ suficiente para evitar entrar en la zona de aumento de extracción o franca anaerobiosis, parece ser 
clave en la prevención del desarrollo del SDMO. En esta primera fase, el $\mathrm{VO}_{2}$ es dependiente del $\mathrm{DO}_{2} \mathrm{y}$ todos los esfuerzos deben estar destinados a aumentar el $\mathrm{DO}_{2}$ mediante el uso de volumen, transfusiones y/o inótropos, para asegurar el $\mathrm{VO}_{2}$ tisular. Sin embargo, de acuerdo a los estudios de Reinhart $^{33}$, Gattinoni ${ }^{28}$ y Hayes ${ }^{34}$ la reanimación tardía de la sepsis no se beneficia de la optimización agresiva del $\mathrm{DO}_{2}$, lo que sugiere la existencia de una segunda fase fisiopatológica en la sepsis, en la cual la optimización del $\mathrm{DO}_{2}$ ya no es capaz de soslayar los daños establecidos en las primeras horas de la sepsis y otros mecanismos patogénicos, no dependientes de variables macro-hemodinámicas de $\mathrm{DO}_{2}$, participan con mayor preponderancia en la génesis del SDMO.

Varios estudios han mostrado que otros factores, distintos al $\mathrm{DO}_{2} \mathrm{y} \mathrm{VO}_{2}$, participan en la génesis del SDMO durante la sepsis y que su manipulación individual o conjunta podría mejorar el pronóstico de esta patología. Entre estos se encuentran la respuesta inmune exacerbada ${ }^{37,38}$ y la posterior inmunosupresión ${ }^{39}$, los trastornos pro-trombóticos ${ }^{40}$ y microcirculatorios ${ }^{41}$, la disfunción mitocondrial o hipoxia citopática ${ }^{42}$, el estrés oxidativo ${ }^{43}$, la hipertensión intraabdominal ${ }^{44}$, entre otros.

\section{Rol de la disfunción mitocondrial en el desarrollo del SDMO}

Evidencias crecientes sugieren que la disfunción mitocondrial tiene un rol central en el SDMO propio de la sepsis ${ }^{45-47}$. La ausencia de muerte celular significativa en la mayoría de los órganos disfuncionantes ${ }^{39,48}$, a excepción del sistema inmune y del epitelio gastrointestinal; la recuperabilidad funcional de los órganos; y la presencia de presiones tisulares de oxígeno normales o incluso elevadas en aquellos órganos disfuncionantes ${ }^{49-54}$, sugieren una disfunción metabólico-celular por sobre una deuda persistente de oxígeno tisular en la sepsis reanimada.

Varios mecanismos participan en la génesis de la disfunción mitocondrial en la sepsis, entre estos destacan: 1) Reducción en la disponibilidad de sustratos como piruvato por bloqueo de la piruvato deshidrogenasa ${ }^{55,56}$, o de NADH por consumo por parte de la enzima poli (ADP-ribosa) polimerasa (PARP-1) $)^{57,58}$; 2) Inhibición directa de los complejos de la cadena de fosforilación oxidativa secun- daria a stress oxidativo ${ }^{43,59,60} ; 3$ ) Disminución en el contenido celular mitocondrial ${ }^{46}$; 4) Aumento en la permeabilidad mitocondrial, ya sea por aumento en la expresión de proteínas desacopladoras o del poro de transición de permeabilidad mitocondrial, ambos fenómenos que se asocian a pérdida del gradiente mitocondrial, caída en la síntesis de ATP y activación de vías de apoptosis.

\section{Efectos deletéreos de la hiper-reanimación}

Como se ha discutido, el $\mathrm{DO}_{2}$ depende fundamentalmente del $\mathrm{GC}$ y del $\mathrm{CaO}_{2}$, la optimización del $\mathrm{DO}_{2}$ en la sepsis implica principalmente el uso de volumen, transfusiones e inotropos, y en la disminución del $\mathrm{VO}_{2}$ mediante el uso de sedación y, si es necesario, ventilación mecánica. El uso de volumen en la sepsis es el tratamiento fundamental para corregir la hipoperfusión global y mejorar el pronóstico de los enfermos, cuando se aporta precozmente y en el contexto de hipoperfusión global $\left(\mathrm{SvO}_{2}<70 \% \text {, lactato }>2,5 \mathrm{mmol} / \mathrm{l}\right)^{27}$. Sin embargo, la optimización del $\mathrm{DO}_{2}$ en la sepsis ya reanimada o estabilizada es de limitado valor terapéutico ${ }^{28}$. Continuar usando grandes cantidades de volumen en pacientes que ya no se benefician de un mayor $\mathrm{DO}_{2}$, lleva a balances hídricos positivos y a mayor edema. Estudios recientes han demostrado que balances hídricos positivos persistentes se correlacionan con más intensa disfunción multiorgánica y con peor pronóstico ${ }^{2,61-67}$. Esto ha sido demostrado para pacientes críticos en general $^{63}$ : sépticos $^{2,67}$, traumatizados ${ }^{68}$, quemados ${ }^{64}$ y con síndrome de distress respiratorio agudo ${ }^{61,62,65}$. Así también, balances hídricos positivos se asocian al desarrollo de hipertensión intraabdominal y síndrome compartimental del abdomen ${ }^{68}$.

\section{Conclusiones}

El conocimiento de la relación $\mathrm{VO}_{2} / \mathrm{DO}_{2}$ es fundamental en el manejo de los enfermos críticos, y en particular de aquellos sépticos. La caída en el $\mathrm{VO}_{2}$ se relaciona a aumento en la mortalidad de los pacientes sépticos ${ }^{69}$, por lo que asegurar un $\mathrm{DO}_{2}$ suficiente para mantener el $\mathrm{VO}_{2}$ tisular mejora el pronóstico de los enfermos. La dependencia $\mathrm{VO}_{2}$ al $\mathrm{DO}_{2}$ predomina en las etapas iniciales de la sepsis como mecanismos fisiopatológico del SDMO. 
En etapas posteriores parecen predominar otros factores, como la respuesta hiper- e hipo-inmune, los trastornos microcirculatorios, la hipoxia citopática, entre otros, como determinantes de falla multiorgánica y mortalidad.

\section{Referencias}

1. Angus DC, Linde-Zwirble WT, Lidicker J, Clermont G, Carcillo J, Pinsky MR. Epidemiology of severe sepsis in the United States: analysis of incidence, outcome, and associated costs of care. Crit Care Med 2001; 29: 1303 10.

2. Vincent JL, Sakr Y, Sprung CL, Ranieri VM, Reinhart K, Gerlach H, et al. Sepsis in European intensive care units: results of the SOAP study. Crit Care Med 2006; 34: 34453.

3. Elbers PW, Ince C. Mechanisms of critical illness-classifying microcirculatory flow abnormalities in distributive shock. Crit Care 2006; 10: 221.

4. Krown KA, Page MT, Nguyen C, Zechner D, Gutiérrez V, Comstock KL et al. Tumor necrosis factor alphainduced apoptosis in cardiac myocytes. Involvement of the sphingolipid signaling cascade in cardiac cell death. J Clin Invest 1996; 98: 2854-65.

5. Abraham E. Neutrophils and acute lung injury. Crit Care Med 2003; 31: S195-9.

6. Booke M, Hinder F, Mcguire R, Traber LD, Traber DL. Nitric oxide synthase inhibition versus norepinephrine for the treatment of hyperdynamic sepsis in sheep. Crit Care Med 1996; 24: 835-44.

7. Whitworth PW, Cryer HM, Garrison RN, Baumgarten TE, Harris PD. Hypoperfusion of the intestinal microcirculation without decreased cardiac output during live Escherichia coli sepsis in rats. Circ Shock 1989; 27: 111-22.

8. Fernandes CJ, JR, Akamine N, Knobel E. Myocardial depression in sepsis. Shock 2008; 30 Suppl 1: 14-7.

9. Cerra FB. The systemic septic response: concepts of pathogenesis. J Trauma 1990; 30: S169-74.

10. Arnold RC, Shapiro NI, Jones AE, Schorr C, Pope J, Casner E, et al. Multi-Center Study of Early Lactate Clearance as a Determinant of Survival in Patients with Presumed Sepsis. Shock 2008;

11. Marecaux G, Pinsky MR, Dupont E, Kahn RJ, Vincent JL. Blood lactate levels are better prognostic indicators than TNF and IL-6 levels in patients with septic shock. Intensive Care Med 1996; 22: 404-8.

12. Gladden LB. Lactate metabolism: a new paradigm for the third millennium. J Physiol 2004; 558: 5-30.
13. Levy B, Sadoune LO, Gelot AM, Bollaert PE, Nabet P, Larcan A. Evolution of lactate/pyruvate and arterial ketone body ratios in the early course of catecholaminetreated septic shock. Crit Care Med 2000; 28: 114-9.

14. Gilbert EM, Haupt MT, Mandanas RY, Huaringa AJ, Carlson RW. The effect of fluid loading, blood transfusion, and catecholamine infusion on oxygen delivery and consumption in patients with sepsis. Am Rev Respir Dis 1986; 134: 873-8.

15. Boekstegers P, Weidenhofer S, Kapsner T, Werdan K. Skeletal muscle partial pressure of oxygen in patients with sepsis. Crit Care Med 1994; 22: 640-50.

16. Hotchkiss RS, Karl IE. Reevaluation of the role of cellular hypoxia and bioenergetic failure in sepsis. Jama 1992; 267: 1503-10.

17. De Backer D, Creteur J, Silva E, Vincent JL. The hepatosplanchnic area is not a common source of lactate in patients with severe sepsis. Crit Care Med 2001; 29: 256-61.

18. Levy B, Desebbe O, Montemont C, Gibot S. Increased aerobic glycolysis through beta2 stimulation is a common mechanism involved in lactate formation during shock states. Shock 2008; 30: 417-21.

19. Levy B, Gibot S, Franck P, Cravoisy A, Bollaert PE. Relation between muscle $\mathrm{Na}+\mathrm{K}+$ ATPase activity and raised lactate concentrations in septic shock: a prospective study. Lancet 2005; 365: 871-5.

20. Kirton OC, Windsor J, Wedderburn R, Hudson-Civetta J, Shatz DV, Mataragas NR, et al. Failure of splanchnic resuscitation in the acutely injured trauma patient correlates with multiple organ system failure and length of stay in the ICU. Chest 1998; 113: 1064-9.

21. Poeze M, Takala J, Greve JW, Ramsay G. Pre-operative tonometry is predictive for mortality and morbidity in high-risk surgical patients. Intensive Care Med 2000; 26 : 1272-81.

22. Haglund U, Bulkley GB, Granger DN. On the pathophysiology of intestinal ischemic injury. Clinical review. Acta Chir Scand 1987; 153: 321-4.

23. Uusaro A, Lahtinen P, Parviainen I, Takala J. Gastric mucosal end-tidal PCO2 difference as a continuous indicator of splanchnic perfusion. Br J Anaesth 2000; 85: 563-9.

24. Gutierrez G, Palizas F, Doglio G, Wainsztein N, Gallesio A, Pacin J, et al. Gastric intramucosal $\mathrm{pH}$ as a therapeutic index of tissue oxygenation in critically ill patients. Lancet 1992; 339: 195-9.

25. Mohsenifar Z, Hay A, Hay J, Lewis MI, Koerner SK. Gastric intramural $\mathrm{pH}$ as a predictor of success or failure in weaning patients from mechanical ventilation. Ann Intern Med 1993; 119: 794-8. 
26. Ivatury RR, Porter JM, Simon RJ, Islam S, John R, Stahl WM. Intra-abdominal hypertension after lifethreatening penetrating abdominal trauma: prophylaxis, incidence, and clinical relevance to gastric mucosal $\mathrm{pH}$ and abdominal compartment syndrome. J Trauma 1998; 44: 1016-21.

27. Rivers E, Nguyen B, Havstad S, Ressler J, Muzzin A, Knoblich B, et al. Early goal-directed therapy in the treatment of severe sepsis and septic shock. N Engl J Med 2001; 345: 1368-77.

28. Gattinoni L, Brazzi L, Pelosi P, Latini R, Tognoni G, Pesenti A, et al. A trial of goal-oriented hemodynamic therapy in critically ill patients. SvO2 Collaborative Group. N Engl J Med 1995; 333: 1025-32.

29. Shoemaker WC, Appel PL, Kram HB, Waxman K, Lee TS. Prospective trial of supranormal values of survivors as therapeutic goals in high-risk surgical patients. Chest 1988; 94: 1176-86.

30. Shoemaker WC, Appel PL, Kram HB. Tissue oxygen debt as a determinant of lethal and nonlethal postoperative organ failure. Crit Care Med 1988; 16: 1117-20.

31. Astiz ME, Rackow EC, Falk JL, Kaufman BS, Weil MH. Oxygen delivery and consumption in patients with hyperdynamic septic shock. Crit Care Med 1987; 15: 26-8.

32. Kaufman BS, Rackow EC, Falk JL. The relationship between oxygen delivery and consumption during fluid resuscitation of hypovolemic and septic shock. Chest 1984; 85: 336-40.

33. Reinhart K, Hannemann L, Kuss B. Optimal oxygen delivery in critically ill patients. Intensive Care Med 1990; 16 Suppl 2: S149-55.

34. Hayes MA, Timmins AC, Yau EH, Palazzo M, Hinds CJ, Watson D. Elevation of systemic oxygen delivery in the treatment of critically ill patients. N Engl J Med 1994; 330: 1717-22.

35. Tuchschmidt J, Fried J, Astiz M, Rackow E. Elevation of cardiac output and oxygen delivery improves outcome in septic shock. Chest 1992; 102: 216-20.

36. Alía I, Esteban A, Gordo F, Lorente JA, Díaz C, Rodríguez $\mathrm{JA}$, et al. A randomized and controlled trial of the effect of treatment aimed at maximizing oxygen delivery in patients with severe sepsis or septic shock. Chest 1999; 115: 453-61.

37. Abraham E, Singer M. Mechanisms of sepsis-induced organ dysfunction. Crit Care Med 2007; 35: 2408-16.

38. Hernández G, Bruhn A, Romero C, Larrondo FJ, De la Fuente R, Cornejo R, et al. Implementation of a norepinephrine-based protocol for management of septic shock: a pilot feasibility study. J Trauma 2006; 60: 77-81.

39. Hotchkiss RS, Swanson PE, Freeman BD, Tinsley KW, Cobb JP, Matuschak GM, et al. Apoptotic cell death in patients with sepsis, shock, and multiple organ dysfunction. Crit Care Med 1999; 27: 1230-51.

40. Bernard GR, Vincent JL, Laterre PF, Larosa SP, Dhainaut JF, López-Rodríguez A, et al. Efficacy and safety of recombinant human activated protein $\mathrm{C}$ for severe sepsis. N Engl J Med 2001; 344: 699-709.

41. De Backer D, Creteur J, Dubois Mj, Sakr Y, Koch M, Verdant C, et al. The effects of dobutamine on microcirculatory alterations in patients with septic shock are independent of its systemic effects. Crit Care Med 2006; 34: 403-8.

42. Fink MP. Bench-to-bedside review: Cytopathic hypoxia. Crit Care 2002; 6: 491-499.

43. Andresen M, Regueira T, Bruhn A, Pérez D, Strobel P, Dougnac A, et al. Lipoperoxidation and protein oxidative damage exhibit different kinetics during septic shock. Mediators Inflamm 2008; 2008: 168652.

44. Regueira T, Bruhn A, Hasbun P, Aguirre M, Romero C, Llanos $\mathrm{O}$, et al. Intra-abdominal hypertension: incidence and association with organ dysfunction during early septic shock. J Crit Care 2008; 23: 461-467.

45. Porta F, Takala J, Weikert C, Bracht H, Kolarova A, Lauterburg $\mathrm{BH}$, et al. Effects of prolonged endotoxemia on liver, skeletal muscle and kidney mitochondrial function. Crit Care 2006; 10: R118.

46. Crouser ED, Julian MW, Huff Je, Struck J, Cook CH. Carbamoyl phosphate synthase-1: a marker of mitochondrial damage and depletion in the liver during sepsis. Crit Care Med 2006; 34: 2439-46.

47. Brealey D, Karyampudi S, Jacques TS, Novelli M, Stidwill $\mathrm{R}$, Taylor V, et al. Mitochondrial dysfunction in a longterm rodent model of sepsis and organ failure. Am J Physiol Regul Integr Comp Physiol 2004; 286: R491-7.

48. Noble JS, Mackirdy FN, Donaldson SI, Howie JC. Renal and respiratory failure in Scottish ICUs. Anaesthesia 2001; 56: 124-9.

49. Rosser DM, Manji M, Cooksley H, Bellingan G. Endotoxin reduces maximal oxygen consumption in hepatocytes independent of any hypoxic insult. Intensive Care Med 1998; 24: 725-9.

50. Rosser DM, Stidwill RP, Jacobson D, Singer M. Oxygen tension in the bladder epithelium rises in both high and low cardiac output endotoxemic sepsis. J Appl Physiol 1995; 79: 1878-82.

51. Dyson A, Stidwill R, Taylor V, Singer M. Tissue oxygen monitoring in rodent models of shock. Am J Physiol Heart Circ Physiol 2007; 293: H526-33.

52. Vandermeer TJ, Wang H, Fink MP. Endotoxemia causes ileal mucosal acidosis in the absence of mucosal hypoxia in a normodynamic porcine model of septic shock. Crit Care Med 1995; 23: 1217-26. 
53. Astiz M, Rackow EC, Weil MH, Schumer W. Early impairment of oxidative metabolism and energy production in severe sepsis. Circ Shock 1988; 26: 311-320.

54. Hotchkiss RS, Rust RS, Dence CS, Wasserman TH, Song SK, Hwang DR, et al. Evaluation of the role of cellular hypoxia in sepsis by the hypoxic marker [18F]fluoromisonidazole. Am J Physiol 1991; 261: R965-972.

55. Vary TC. Increased pyruvate dehydrogenase kinase activity in response to sepsis. Am J Physiol 1991; 260: E669-674.

56. Vary TC, Siegel JH, Nakatani T, Sato T, Aoyama H. Effect of sepsis on activity of pyruvate dehydrogenase complex in skeletal muscle and liver. Am J Physiol 1986; 250: E634-640.

57. Szabo C. Poly (ADP-ribose) polymerase activation and circulatory shock. Novartis Found Symp 2007; 280: 92103; discussion 103-107, 160-104.

58. Khan AU, Delude RL, Han YY, Sappington PL, Han X, Carcillo JA, et al. Liposomal NAD $(+)$ prevents diminished $\mathrm{O}(2)$ consumption by immunostimulated Caco-2 cells. Am J Physiol Lung Cell Mol Physiol 2002; 282: L1082-91.

59. Radi R, Rodríguez M, Castro L, Telleri R. Inhibition of mitochondrial electron transport by peroxynitrite. Arch Biochem Biophys 1994; 308: 89-95.

60. Bolanos JP, Heales SJ, Peuchen S, Barker JE, Land JM, Clark JB. Nitric oxide-mediated mitochondrial damage: a potential neuroprotective role for glutathione. Free Radic Biol Med 1996; 21: 995-1001.

61. Sakr Y, Vincent JL, Reinhart K, Groeneveld J, Michalopoulos A, Sprung CL, et al. High tidal volume and positive fluid balance are associated with worse outcome in acute lung injury. Chest 2005; 128: 3098-108.

62. Schuller D, Mitchell JP, Calandrino FS, Schuster DP. Fluid balance during pulmonary edema. Is fluid gain a marker or a cause of poor outcome? Chest 1991; 100: 1068-75.

63. Sakka SG, Klein M, Reinhart K, Meier-Hellmann A. Prognostic value of extravascular lung water in critically ill patients. Chest 2002; 122: 2080-6.

64. Klein MB, Hayden D, Elson C, Nathens AB, Gamelli RL, Gibran NS, et al. The association between fluid administration and outcome following major burn: a multicenter study. Ann Surg 2007; 245: 622-8.

65. Wiedemann HP, Wheeler AP, Bernard GR, Thompson BT, Hayden D, Deboisblanc B, et al. Comparison of two fluid-management strategies in acute lung injury. $\mathrm{N}$ Engl J Med 2006; 354: 2564-75.

66. Arlati S, Storti E, Pradella V, Bucci L, Vitolo A, Pulici M. Decreased fluid volume to reduce organ damage: a new approach to burn shock resuscitation? A preliminary study. Resuscitation 2007; 72: 371-8.

67. Alsous. Negtive fluid balance. chest 2000; 117: 1749.

68. Balogh Z, Mckinley BA, Cocanour CS, Kozar RA, Valdivia A, Sailors RM, et al. Supranormal trauma resuscitation causes more cases of abdominal compartment syndrome. Arch Surg 2003; 138: 637-42; discussion 642-33.

69. Squara P, Journois D, Formela JF, Schremmer B, Dhainaut JF, Bleichner G. Value of elementary, combined, and modeled hemodynamic variables. J Crit Care 1994; 9: 223-235. 